\title{
The Zeiss-MKM system for frameless image-guided approach in epidural motor cortex stimulation for central neuropathic pain
}

\author{
Benoit Pirotte, M.D., PhilipPe Voordecker, M.D., Freddy Joffroy, M.D., \\ Nicolas Massager, M.D., DaVid Wikler, Ms, Danielle BaleriauX, M.D., \\ Marc Levivier, M.D., Ph.D., ANd Jacques Brotchi, M.D., Ph.D. \\ Departments of Neurosurgery and Neuroradiology, Erasme Hospital, University of Brussels, Belgium
}

\begin{abstract}
Object. Twelve patients (seven female, and five male, mean age 55.6 years) suffering from refractory central (ischemic/traumatic [eight cases]) and neuropathic pain (trigeminal neuropathy [four cases]) underwent surgery for the implantation of an epidural motor cortex stimulation (MCS) device in which the authors used a frameless neuronavigation system, the Zeiss-MKM microscope.

Methods. The authors assessed the spatial accuracy of the neuronavigation system and its potential contribution to improve the quality of targeting pain. In these patients, the positions of the central sulcus, defined by stereotactic magnetic resonance MR imaging, intraoperative somatosensory evoked potentials (SSEPs) and subdural visual verification, were correlated into the stereotactic neuronavigation planning procedure. The mean spatial accuracy of distance between (MR) imaging-defined and actual central sulcus was $2.4 \mathrm{~mm}$ (range 5-10 mm). The intraoperative SSEPdefined central sulcus was close to that defined by MR imaging (mean distance $6.4 \mathrm{~mm}$ ). Although very precise, intraoperative SSEP recordings were impaired by artifacts and wave attenuation in six of the 12 patients. Stereotactic correlations between anatomical and functional data in the navigation system corrected final targeting in 10 of 12 cases. Pain relief was obtained in eight patients. Indeed, inappropriate targeting probably explains the reported variable success rate of MCS and certainly underestimates the actual efficacy.

Conclusions. Since intraoperative SSEP monitoring has, for many years, been considered the standard procedure to approach motor target, the development of an accurate stereotactic image guidance system could help to increase the efficacy of MCS on the alleviation of pain. The excellent spatial accuracy provided by the Zeiss-MKM navigation system allows precise data correlations that represent a remarkable means to validate functional MR imaging as an alternative to SSEP. The authors believe that developing stereotactic image guidance with such a navigation system could improve the success rate of MCS.
\end{abstract}

\section{KEY WORDS • pain $\bullet$ motor cortex stimulation $\bullet$ stereotaxy $\bullet$ neuronavigation}

The actual efficacy of epidural chronic MCS in the alleviation of refractory central and neuropathic pain is still being evaluated. Indeed, different success rates of longterm pain relief ranging from 45 to $75 \%$ have been reported, mainly in chronic pain syndromes related to central poststroke pain or trigeminal neuropathy. ${ }^{1-4,7,18,20,21,24,32-34}$ These variable results could be related to inaccurate positioning of the stimulation electrode, and the actual efficacy of MCS could be underestimated. $3,13,14,16,18,20,32-34$

Since first described, intraoperative epidural SSEP recording became the standard procedure to localize the functional central sulcus and motor target. ${ }^{33,34}$ The surgical approach consisting initially in a trephination in the "estimated motor area" $7,33,34$ was even reduced to a burr

Abbreviations used in this paper: $\mathrm{ES}=$ electrical stimulation; $\mathrm{fMR}=$ functional magnetic resonance; $\mathrm{MCS}=$ motor cortex stimulation; SSEP = somatosensory evoked potential; $3 \mathrm{D}=$ three-dimensional. hole. ${ }^{12,13,18}$ A stereotactic neuronavigator with superficial computerized tomography reconstruction was later used. ${ }^{21-23}$

Because appropriate targeting seems to be a crucial step in efforts to obtain pain relief in afflicted individuals, we developed a procedure in which an MCS device is implanted using a stereotactic image guidance frameless navigation system, the Zeiss-MKM microscope (Carl Zeiss, Oberkochen, Germany). In this preliminary series of 12 patients, our aim was to assess the spatial accuracy of this technique and to emphasize its potential use to improve the efficacy of MCS.

\section{CLINICAL MATERIAL AND METHODS}

\section{Patient Population and Pain Assessment}

Twelve patients (seven women and five men, who ranged in age from 33 to 70 years [mean 55.6 years]), suf- 
fering from a chronic pain syndrome secondary to central or neuropathic lesions, have been treated since 1997 by chronic stimulation of the motor cortex. The underlying lesion and somatic distribution of pain are summarized in Table 1. Pain was refractory to extensive oral or intrathecal pharmacotherapy, and some patients had undergone different neurosurgical procedures to obtain resolution of symptoms. All patients gave informed consent to the procedure and were treated according to the ethical guidelines of our institution. The pain and changes in pain level were assessed by a neurologist and a psychiatrist of the Pain Clinic. Pain level was preoperatively evaluated using a visual analog scale with scores ranging from 0 to 100 . The efficacy of MCS to reduce pain was classified into four previously described categories: 1) excellent (80-100\% pain reduction); 2) good (60-79\% improvement); 3) satisfactory (40-59\% improvement); and 4$)$ failure $(<40 \%$ improvement). ${ }^{9,13,20,21,24,32-34}$

\section{Stereotactic Image Guidance and Surgical Procedure}

We applied a technique combining the intraoperative epidural SSEP recordings with stereotactic image guidance. ${ }^{20}$ We used a frameless neuronavigation system, the Zeiss-MKM microscope, and acquired MR imaging data in frameless stereotactic conditions with the skin-based markers. Axial 3D T $\mathrm{T}_{1}$-weighted MR images (130 slices) of the brain were transferred into a stereotaxis planning workstation (STP4.0; Leibinger/Fischer, Freiburg, Germany), allowing multiplanar visualization of the brain structures, especially the cortical sulci. The central sulcus was localized ${ }^{36}$ and its precise shape registered in the 3D planning workstation (Fig. 1). The vertical projection of the insular cistern on the central sulcus was estimated to correspond grossly to the motor target of the hand and was projected on the skin surface by the Zeiss-MKM microscope to center the craniectomy. A $5 \times 5-\mathrm{cm}$-square craniectomy was performed after induction of general anesthesia with propofol and sufentanil. The patient's head was fixed in a Mayfield clamp to allow for stereotactic navigation.

A quadripolar electrode (Resume II; Medtronic Inc., Minneapolis, MN) was placed at different locations on the dural surface to cover the central sulcus region. The location of the central sulcus was defined by means of the phase reversal of the $\mathrm{N}_{20} \mathrm{P}_{30}$ wave on the intraoperative SSEP after stimulation of the contralateral median nerve, as has been previously described. ${ }^{37}$ Stimulation of some areas of the contralateral side of the face was performed in patients with facial pain, according to a previously described technique. ${ }^{17}$ The location of the motor cortex was again confirmed by ES of the cortex through the Resume II electrode (pulse duration $1 \mathrm{msec}, 5-\mathrm{Hz}$ trains) in the last five patients to undergo the procedure. We used a Pathfinder Viking IV stimulator (Nicolet Biomedical Inc., Madison, WI). With this method, an epidural map of the functional central sulcus was designed by intraoperative SSEP as previously described. ${ }^{20,21,37}$ After definition of the functional central sulcus by intraoperative SSEP, spatial coordinates of the intraoperative SSEP-defined central sulcus and motor target were projected on the stereotactic navigation planning station. Before fixating the stimulation electrode epidurally, the dura mater was opened and immediately sutured (without perforation of the arachnoid layer) to abolish local stimulation pain. ${ }^{20,21,32}$ The actual coordinates of the central sulcus were then visually verified and registered using the Zeiss-MKM microscope.

In all patients, fMR imaging was also performed. Our intention was only to assess its feasibility and to compare its results with data obtained by the standard localizing technique.

\section{Stereotactic Correlations}

At this stage of the procedure, we registered and correlated in the stereotactic navigation planning system all the targets obtained by anatomical imaging and observation

TABLE 1

Summary of neurological data in 12 patients with refractory chronic pain syndromes*

\begin{tabular}{|c|c|c|c|c|c|c|c|}
\hline \multirow[b]{3}{*}{$\begin{array}{l}\text { Case } \\
\text { No. }\end{array}$} & \multirow[b]{3}{*}{$\begin{array}{l}\text { Age (yrs), } \\
\text { Sex }\end{array}$} & \multirow[b]{3}{*}{$\begin{array}{c}\text { Pain } \\
\text { Territory }\end{array}$} & \multirow[b]{3}{*}{$\begin{array}{c}\text { Underlying } \\
\text { Lesion }\end{array}$} & \multirow[b]{3}{*}{$\begin{array}{c}\text { Unsuccessful } \\
\text { Therapies }\end{array}$} & \multicolumn{3}{|c|}{ Disturbances } \\
\hline & & & & & \multicolumn{2}{|c|}{ Sensibility } & \multirow{2}{*}{$\begin{array}{c}\text { Motor } \\
\text { Voluntary } \\
\text { Movements }\end{array}$} \\
\hline & & & & & $\begin{array}{c}\text { Hypes- } \\
\text { thesia }\end{array}$ & $\begin{array}{l}\text { Allo- } \\
\text { dynia }\end{array}$ & \\
\hline 1 & $40, \mathrm{~F}$ & face $\left(V^{2-3}\right)$ & trigeminal neuralgia & TC, BMC, MVD & + & + & normal \\
\hline 2 & $62, \mathrm{M}$ & upper limb & capsular stroke & NSAID, TR, BZD & ++ & + & paralysis \\
\hline 3 & $65, \mathrm{~F}$ & lower limbs & spinal cord ependymoma & ITB, ITM, SCS & + & ++ & paresis \\
\hline 4 & $33, \mathrm{~F}$ & upper limb & brainstem stroke & NSAID, TR, BZD & + & + & normal \\
\hline 5 & $66, \mathrm{~F}$ & upper limb & subcortical Stroke & NSAID, TR, BZD & + & + & paresis \\
\hline 6 & $34, \mathrm{M}$ & upper limb & plexus avulsion & NSAID, TR, BZD, TENS & + & ++ & paralysis \\
\hline 7 & $38, \mathrm{~F}$ & face $\left(V^{2}\right)$ & dental avulsion & $\mathrm{TC}, \mathrm{CBZ}$ & + & +++ & normal \\
\hline 8 & $70, \mathrm{M}$ & upper limb & cervical syrinx & syrinx drainage & + & + & paresis \\
\hline 9 & $65, \mathrm{~F}$ & upper limb & postradic plexopathy & NSAID, TR, BZD, TENS & +++ & +++ & paralysis \\
\hline 10 & $50, \mathrm{~F}$ & face $\left(V^{2-3}\right)$ & multiple sclerosis & NSAID, TR, BZD, CBZ & + & ++ & normal \\
\hline 11 & $73, \mathrm{M}$ & upper limb & arm avulsion/amputation & NSAID, TR, BZD & +++ & + & none \\
\hline 12 & $70, \mathrm{M}$ & face $\left(V^{2}\right)$ & tumor & RT, TR, CBZ, BZD & + & ++ & normal \\
\hline
\end{tabular}

$* \mathrm{BMC}=$ balloon microcompression; $\mathrm{BZD}=$ benzodiazepines $; \mathrm{CBZ}=$ carbamazepine ITB $=$ intrathecal baclofen; ITM = intrathecal morphine; MVD = microvascular decompression; NSAID = nonsteroidal antiinflammatory drugs; RT = radiation therapy; SCS = spinal cord stimulation; TENS = transcutaneous electrical nerve stimulation; TC = thermocoagulation; TR = tricyclic antidepressants; $\mathrm{V}^{2}, \mathrm{~V}^{3}=$ second and third branches of the trigeminal nerve; $+=$ moderate; $++=$ severe $;+++=$ maximum. 


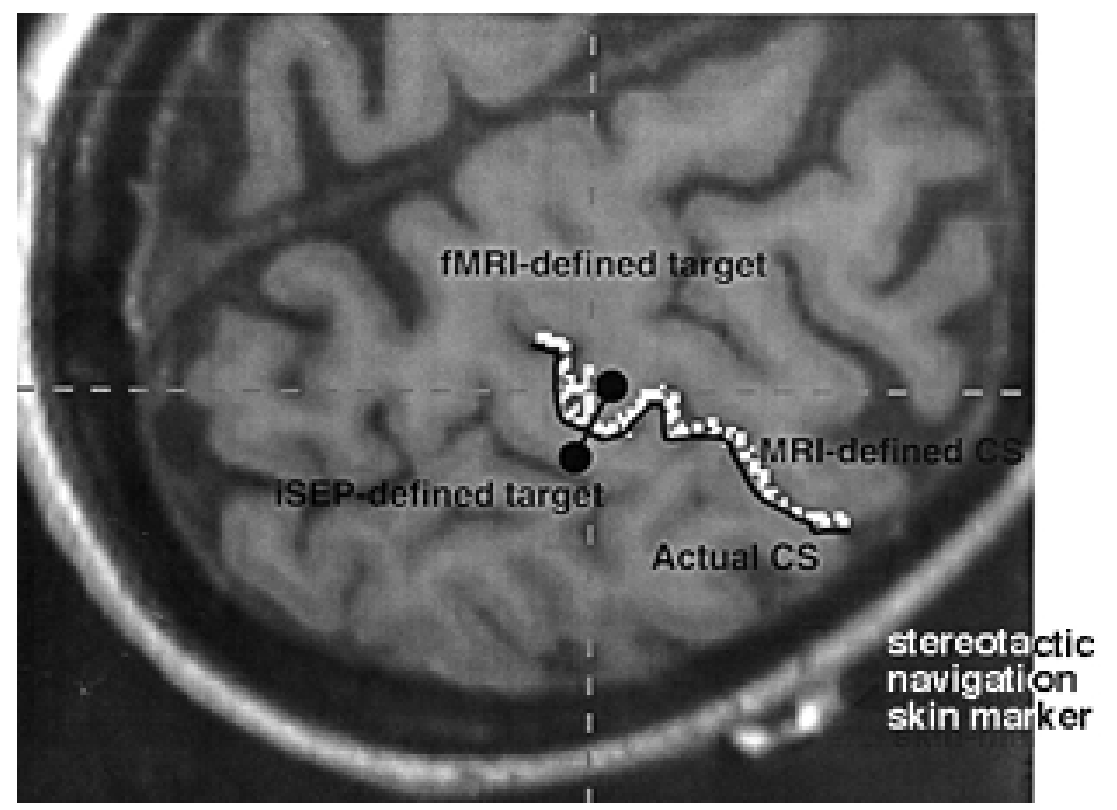

Fig. 1. Oblique view of a multiplanar MR imaging-based navigation planning image pictured on the stereotactic workstation (note the stereotactic navigation skin marker). The image shows the spatial correlation between targets obtained from different anatomical and functional methods: the MR imaging-defined central sulcus (central sulcus), the actual central sulcus after subdural visual verification (white), the motor target of the hand defined by intraoperative SSEP recordings (SSEP-defined motor target), and the motor target of the hand defined by fMR imaging (fMR imagingdefined motor target).

(that is, MR imaging-defined central sulcus and actual central sulcus landmarks) and functional recordings (intraoperative SSEP-defined central sulcus and motor target). We measured, in the sagittal plane, the distance between the MR imaging-defined central sulcus and the actual central sulcus to verify the spatial accuracy provided by the navigation system. The operative shift of the brain structures should not be significant because the surgical procedure is epidural (at least totally extraarachnoid). We also measured, in the sagittal plane, the distance between MR imaging-defined and intraoperative SSEPdefined central sulcus to assess the precision of the latter techinique's localizing accuracy.

\section{Stimulation Procedure}

Once the motor target was identified, the Resume II stimulation electrode was fixated epidurally, as previously described. ${ }^{20}$ During the 4 -week period in which the stimulation trial took place, the motor cortex in each patient received stimulation for 1 hour every 4 hours during the day with an external stimulator (monophasic square wave pulses; frequency $40 \mathrm{~Hz}$, duration $100 \mu \mathrm{sec}$, amplitude 1-5 V). Many bipolar combinations were tested with the negative pole situated over the motor cortex. Pain and changes in pain level were assessed as described previously. The delay between the start of stimulation and the onset of pain alleviation was noted as was the duration of pain relief after the ES was switched off (Table 2). Those patients in whom excellent and good responses to stimulation were obtained subsequently underwent implantation of the MCS system (Itrel 3; Medtronic Inc., Minneapolis, MN) for long-term stimulation.

\section{RESULTS}

\section{Analgesic Efficacy}

Motor cortex stimulation induced significant, reproducible, and long-lasting pain relief in eight patients (excellent reduction in six [Cases 2, 4, 5, and 10-12] and good relief in two [Cases 1 and 8]; Table 2). The alleviation of pain occurred from 10 to 15 minutes after the start of stimulation and lasted from 15 to 120 minutes after the 1-hour duration of ES was switched off. Two patients reported that pain relief remained stable for more than 24 hours if the stimulation period was longer than 4 hours. Moreover, the first three patients in whom the MCS system was implanted described that severe pain recurred when they switched the ES off for more than 2 days. In four patients (Cases 3, 6, 7, and 9) no response to MCS was elicited. In one patient (Case 12), a 10-minute ES period at low amplitude $(<1 \mathrm{~V})$, induced focal seizures, although it was associated with excellent pain relief. In four patients (Case $3,6,7$, and 9), no analgesic effect was observed. The patients in Cases 6 and 9 were those in whom the most significant wave attenuation was observed on intraoperative SSEP recordings. In these cases, repeated intraoperative SSEP recordings provided inconsistent reproducibility and difficult definition of the final motor target. After the failure was observed, we proposed reoperation to both patients (with intraoperative SSEP recordings) for repositioning the electrode. The patient in Case 6 agreed to undergo the procedure, but repositioning did not yield clinical effect. The patient with refractory lower-extremity pain (Case 3) experienced no clinical effect. Finally, although MCS failed to alleviate pain, no additional surgery was performed in the patient in Case 7 because operative 
TABLE 2

Effect of MCS on alleviation of pain*

\begin{tabular}{|c|c|c|c|c|c|c|c|}
\hline \multirow{3}{*}{$\begin{array}{l}\text { Case } \\
\text { No. }\end{array}$} & \multirow{2}{*}{\multicolumn{2}{|c|}{ Analgesic Effect }} & \multicolumn{3}{|c|}{ Stimulation } & \multirow{3}{*}{$\begin{array}{l}\text { Intake of An- } \\
\text { algesic Drugs }\end{array}$} & \multirow{3}{*}{$\begin{array}{c}\text { Induced Paresthesia } \\
\text { or Movements }\end{array}$} \\
\hline & & & \multirow{2}{*}{$\begin{array}{l}\text { Duration } \\
\text { (hrs) }\end{array}$} & \multirow{2}{*}{$\begin{array}{l}\text { Delay Before } \\
\text { Effect (mins) }\end{array}$} & \multirow{2}{*}{$\begin{array}{l}\text { Duration of } \\
\text { Effect (mins) }\end{array}$} & & \\
\hline & VAS & Result & & & & & \\
\hline 1 & $7>2$ & good & $1 / 4$ & 10 & $15-20$ & not reduced & none \\
\hline 2 & $6>1$ & excellent & $2 / 3$ & 15 & 30 & reduced by $50 \%$ & none \\
\hline 3 & $7>6$ & failure & & & & & \\
\hline 4 & $8>1$ & excellent & $2 / 4$ & 15 & 45 & reduced by $75 \%$ & none \\
\hline 5 & $7>0$ & excellent & $1 / 3$ & 10 & 60 & reduced by $50 \%$ & none \\
\hline 6 & $8>8$ & failure & & & & & \\
\hline 7 & $8>7$ & failure & & & & & \\
\hline 8 & $7>2$ & good & $1 / 4$ & 10 & 60 & reduced by $50 \%$ & none \\
\hline 9 & $7>7$ & failure & & & & & \\
\hline 10 & $8>0$ & excellent & $1 / 6$ & 10 & 120 & reduced by $75 \%$ & \\
\hline 11 & $8>1$ & excellent & $2 / 3$ & 15 & 30 & reduced by $50 \%$ & \\
\hline 12 & $7>1$ & excellent & $1 / 4$ & 15 & 45 & not reduced & yes \\
\hline
\end{tabular}

* The VAS graduated from 0 to 100 . The analgesic efficacy of MCS was classified into 4 categories: 1$)$ excellent ( 80 to $100 \%$ pain reduction); 2) good (60 to $79 \%$ improvement); 3 ) satisfactory (40 to $59 \%$ improvement); 4 ) failure (<40\% improvement). The duration of stimulation is given as the lowest ratio between the length of stimulation period and duration separating the start of the two stimulation cycles.

data were precise and concordant and no alternative target could be proposed.

\section{Stereotactic Image Guidance and Surgical Procedure}

Visualization of cortical sulci on stereotactic MR imaging was achieved in all patients (Table 3 ). In patients in Cases 1, 6, 7, 9, 11, and 12, the quality of intraoperative $\mathrm{SSEP}$ recordings was not optimal. In the patient in Case 1, because intense artifacts related to the operating room environment were present, reoperation was performed several days later in another room, which was thereafter reserved for future MCS procedures. A severe wave attenuation, due to the underlying lesion of the somatosensory tract, was observed in three patients (Cases 6, 9, and 11). No clear evoked potential was observed on facial stimulation in two patients (Cases 7 and 12). Epidural bipolar motor stimulation also performed in the last five patients to be treated yields little additional localizing information because diffuse muscle contractions were clinically visualized even after low-amplitude stimulation on the parietal cortex. We observed no complication, discomfort, computer failure, or mechanical problem related to the use of the navigation system. Finally, one patient (Case 11) developed a subdural infection that required the removal of the implanted electrode.

\section{Stereotactic Correlations}

The mean distance between the MR imaging-defined and actual central sulcus was $2.4 \mathrm{~mm}$, and between the MR imaging-defined and intraoperative SSEP-defined central sulcus it was $6.4 \mathrm{~mm}$ (Table 3 ). These correlations are detailed in the Discussion.

\section{DISCUSSION}

This preliminary series represents the first step of a prospective study of MCS in patients with refractory central and neuropathic pain. The following results were obtained: 1) confirmation of the clinical efficacy of MCS; 2) emphasis of the usefulness of a stereotactic image guidance in MCS; 3) confirmation of the excellent spatial accuracy of the Zeiss-MKM microscope; and 4) presentation of the interest of stereotactic data correlations.

\section{Clinical Efficacy}

Motor cortex stimulation induced complete pain relief in six $(50 \%)$ of the 12 patients and significant but incomplete pain relief in two others. This effect was reproducible and persistent on long-term follow up. We observed that the analgesic effect occurred a few minutes after stimulation. It also remained stable for several minutes after the ES was discontinued. This small series does not allow us to discuss the results according to the type and location of the underlying lesion. Our results can be compared with

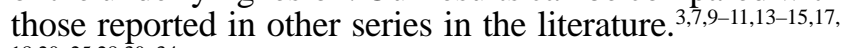
${ }_{18,20-25,28,30-34}$ Various success rates of long-term pain relief, however, ranging from 45 to $75 \%$, have been reported. 3,7 , 18,20,21,24,32-34 The best results were obtained in patients with chronic painful syndromes due to central post-stroke pain and trigeminal neuropathy. ${ }^{7,18,21,32}$ The variability of these results could be related to multiple factors including the quality of patient selection. Different techniques have been proposed to improve the quality of patient selection, including barbiturates an morphine test ${ }^{35}$ and the observation that transcranial magnetic coil stimulation of the motor cortex could predict the response to epidural MCS in patients with deafferentation pain. ${ }^{5,6,19}$ This suggests that a better patient selection process could improve the MCS success rate. ${ }^{10,11,15,25,35}$

In the assessment period of the four patients in whom no response to MCS was demonstrated, we also wondered about the inaccuracy of targeting. The variability of the reported results could also be related to inaccurate positioning of the stimulation electrode. ${ }^{18,33}$ Because appropriate targeting is a crucial step in the effort to induce pain relief, the actual efficacy of MCS could be underestimated. This possibility led us to the conviction that the optimization of MCS efficacy will require the development of an accurate stereotactic image guidance system. ${ }^{22,23,29}$ 
TABLE 3

Technical problems and characteristics*

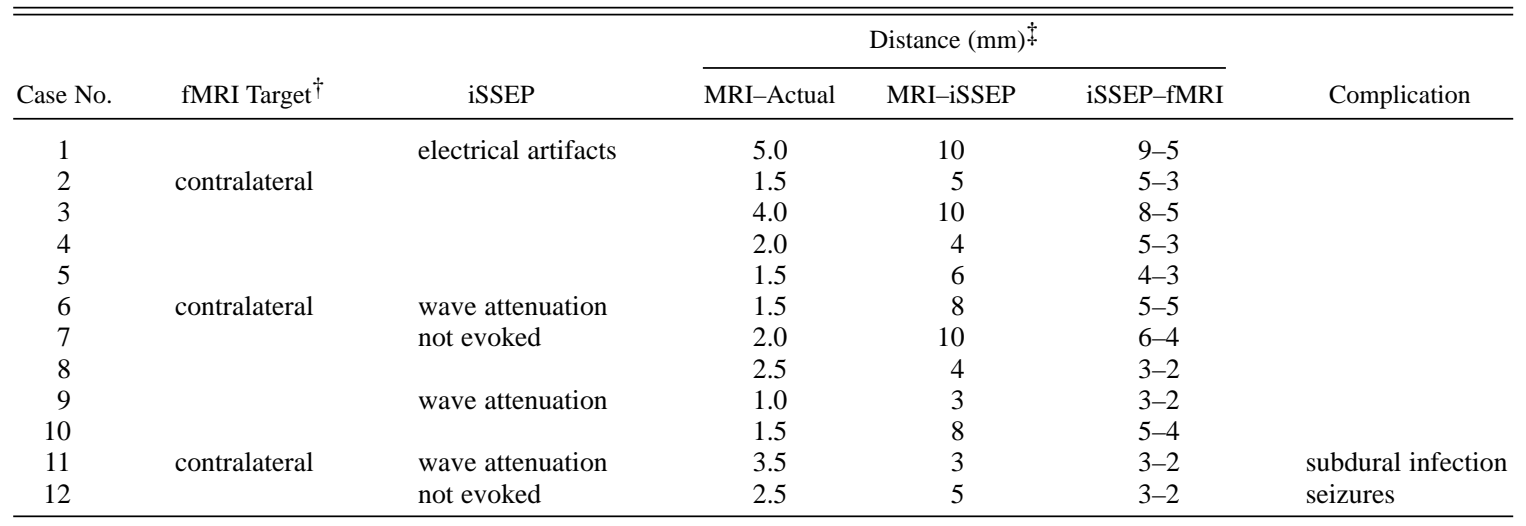

* $\mathrm{fMRI}=$ functional MR imaging; iSSEP $=$ intraoperative SSEP.

$\dagger$ Functional MR imaging was used to study the movement of the hand contralateral to the painful area.

$\neq$ MRI-Actual = distance between MR imaging-defined central sulcus and actual position of the central sulcus after subdural visual verification; MRI-iSSEP = distance between MR imaging-defined central sulcus and intraoperative SSEP-defined central sulcus; iSSEP-fMRI: distance between intraoperative SSEP-defined motor target and fMR imaging-defined motor target.

\section{Stereotactic Image Guidance}

This preliminary experience confirms the potential role of stereotactic image guidance in MCS. Although intraoperative SSEP recordings had early become the standard procedure to localize the functional central sulcus and motor target, ${ }^{7,18,28,33}$ the surgical approach was initially limited to a trephination or a burr hole in the "estimated motor area"7,12,13,18,33,34 to reduce the invasiveness of the technique. A simple procedure involving a 3D MR image with an external reference grid was proposed to define the central area grossly. ${ }^{12}$ Nguyen and coworkers ${ }^{20-24}$ were the first to use a stereotactic neuronavigator with superficial computerized tomography reconstruction to develop the technique further.

The Zeiss-MKM microscope is a commercially available frameless navigation system. From a surgical point of view, a large bone opening, skin incision, and shaving of the scalp appeared less important to these disabled patients than the actual therapeutic challenge of pain. Technically, the method is minimally invasive as long as the procedure remains epidural. In our experience, there is no need to implicate the epidural site of the stimulation electrode as a cause of the clinical failure; when no analgesic effect is obtained by stimulation of more than $6 \mathrm{~V}$, there is no benefit to increasing the amplitude or placing the electrode subdurally.

\section{Spatial Accuracy of the Navigation System and Interest of Stereotactic Data Correlations}

These preliminary results show the excellent spatial accuracy obtainable when using the Zeiss-MKM microscope, which was compatible with the degree of precision required for MCS. This led us to consider the navigation planning system as a remarkable tool that allows spatial correlation between targets from different anatomical and functional methods.

First, the correlation between the MR imaging- and intraoperative SSEP-defined central sulcus data confirmed the high precision of latter to localize the central sul- cus. $^{26,27,36}$ Unfortunately, the correlation of data obtained using these modalities revealed the practical limitations of intraoperative SSEP recordings, thus indicating the potential superiority of fMR imaging as a localizing technique in this indication. Indeed, in all patients fMR imaging was also performed. Our goal was only to assess its feasibility. In this preliminary study, the fMR imaging-defined motor target was not considered for guiding MCS placement. We introduced the fMR imaging sequences in the stereotactic navigation planning, ${ }^{29}$ however, and compared the results with data obtained using intraoperative SSEP (Fig. 1).

During the navigation planning, we measured vertically and horizontally, in the sagittal plane, the distance between the intraoperative SSEP-defined motor target and the center of the fMR imaging-defined target of the hand. The mean distance between intraoperative SSEP- and fMR imaging-defined precentral activation signal was always vertically inferior to $9 \mathrm{~mm}$ and anteroposteriorly inferior to $5 \mathrm{~mm}$. These correlations illustrated the precision of localizing the functional central sulcus in all cases. Functional MR imaging always provided a highly reproducible focal target diameter ranging from 5 to $10 \mathrm{~mm}$ whereas the quality of intraoperative SSEP recordings presented some limitations in $50 \%$ of patients. Additionally, in 10 of 12 patients (Cases 1, 2, 4-6, and 8-12), the correlations between the intraoperative SSEP- and fMR imaging-defined motor targets could correct the targeting, either confirming the intraoperative SSEP-defined targeting or helping to localize the target precisely obtained by artifacted/attenuated intraoperative SSEP recordings. These last two points will be developed in another work in which the feasibility of using fMR imaging as an alternative to intraoperative SSEP monitoring is assessed.

\section{CONCLUSIONS}

We believe that the use of a stereotactic image guidance could improve the success rate of MCS in the alleviation of central neuropathic pain. The navigation system of the Zeiss-MKM microscope provided excellent spatial accu- 
racy that allowed precise correlations of data obtained using different techniques. These correlations helped to correct the final targeting and allowed prospective validation of new techniques such as fMR imaging.

\section{References}

1. Canavero S, Bonicalzi V: Cortical stimulation for central pain. J Neurosurg 83:1117, 1995

2. Canavero S, Bonicalzi V, Castellano G, et al: Painful supernumerary phantom arm following motor cortex stimulation for central poststroke pain. Case report. J Neurosurg 91:121-123, 1999

3. Carroll D, Joint C, Maartens N, et al: Motor cortex stimulation for chronic neuropathic pain: a preliminary study of 10 cases. Pain 84:431-437, 2000

4. Davis KD, Lozano AM, Tasker RR, et al: Brain targets for pain control. Stereotact Funct Neurosurg 71:173-179, 1998

5. Di Lazzaro V, Oliviero A, Profice P, et al: Direct demonstration of interhemispheric inhibition of the human motor cortex produced by transcranial magnetic stimulation. Exp Brain Res 124:520-524, 1999

6. Di Lazzaro V, Restuccia D, Oliviero A, et al: Magnetic transcranial stimulation at intensities below active motor threshold activates intracortical inhibitory circuits. Exp Brain Res 119: 265-268, 1998

7. Ebel H, Rust D, Tronnier V, et al: Chronic precentral stimulation in trigeminal neuropathic pain. Acta Neurochir 138: 1300-1306, 1996

8. Franzini A, Ferroli P, Servello D, et al: Reversal of thalamic hand syndrome by long-term motor cortex stimulation. J Neurosurg 93:873-875, 2000

9. Fujii M, Ohmoto Y, Kitahara T, et al: [Motor cortex stimulation therapy in patients with thalamic pain.] No Shinkei Geka 25: 315-319, 1997 (Jpn)

10. Garcia-Larrea L, Peyron R, Mertens P, et al: Electrical stimulation of motor cortex for pain control: a combined PET-scan and electrophysiological study. Pain 83:259-273, 1999

11. Garcia-Larrea L, Peyron R, Mertens P, et al: Positron emission tomography during motor cortex stimulation for pain control. Stereotact Funct Neurosurg 68:141-148, 1997

12. Herregodts P, Stadnik T, De Ridder F, et al: Cortical stimulation for central neuropathic pain: 3-D surface MRI for easy determination of the motor cortex. Acta Neurochir Suppl 64: 132-135, 1995

13. Hosobuchi Y: Motor cortical stimulation for control of central deafferentation pain. Adv Neurol 63:215-217, 1993

14. Katayama Y, Fukaya C, Yamamoto T: Control of poststroke involuntary and voluntary movement disorders with deep brain or epidural cortical stimulation. Stereotact Funct Neurosurg 69:73-79, 1997

15. Katayama Y, Fukaya C, Yamamoto T: Poststroke pain control by chronic motor cortex stimulation: neurological characteristics predicting a favorable response. J Neurosurg 89:585-591, 1998

16. Katayama Y, Tsubokawa T, Yamamoto T: Chronic motor cortex stimulation for central deafferentation pain: experience with bulbar pain secondary to Wallenberg syndrome. Stereotact Funct Neurosurg 62:295-299, 1994

17. McCarthy G, Allison T, Spencer DD: Localization of the face area of human sensorimotor cortex by intracranial recording of somatosensory evoked potentials. J Neurosurg 79:874-884, 1993

18. Meyerson BA, Lindblom U, Linderoth B, et al: Motor cortex stimulation as treatment of trigeminal neuropathic pain. Acta Neurochir Suppl 58:150-153, 1993

19. Migita K, Uozumi T, Arita K, et al: Transcranial magnetic coil stimulation of motor cortex in patients with central pain. Neurosurgery 36:1037-1040, 1995
20. Nguyen JP, Keravel Y, Feve A, et al: Treatment of deafferentation pain by chronic stimulation of the motor cortex: report of a series of 20 cases. Acta Neurochir Suppl 68:54-60, 1997

21. Nguyen JP, Lefaucheur JP, Decq P, et al: Chronic motor cortex stimulation in the treatment of central and neuropathic pain. Correlations between clinical, electrophysiological and anatomical data. Pain 82:245-251, 1999

22. Nguyen JP, Lefaucheur JP, Le Guerinel C, et al: Motor cortex stimulation in the treatment of central and neuropathic pain. Arch Med Res 31:263-265, 2000

23. Nguyen JP, Lefaucheur JP, Le Guerinel C, et al: [Treatment of central and neuropathic facial pain by chronic stimulation of the motor cortex: value of neuronavigation guidance systems for the localization of the motor cortex.] Neurochirurgie 46: 483-491, 2000 (Fr)

24. Nguyen JP, Pollin B, Feve A, et al: Improvement of action tremor by chronic cortical stimulation. Mov Disord 13:84-88, 1998

25. Peyron R, Garcia-Larrea L, Deiber MP, et al: Electrical stimulation of precentral cortical area in the treatment of central pain: electrophysiological and PET study. Pain 62:275-286, 1995

26. Puce A, Constable RT, Luby ML, et al: Functional magnetic resonance imaging of sensory and motor cortex: comparison with electrophysiological localization. J Neurosurg 83: 262-270, 1995

27. Pujol J, Conesa G, Deus J, et al: Presurgical identification of the primary sensorimotor cortex by functional magnetic resonance imaging. J Neurosurg 84:7-13, 1996

28. Rainov NG, Fels C, Heidecke V, et al: Epidural electrical stimulation of the motor cortex in patients with facial neuralgia. Clin Neurol Neurosurg 99:205-209, 1997

29. Roux FE, Ibarrola D, Lazorthes Y, et al: Chronic motor cortex stimulation for phantom limb pain: a functional magnetic resonance imaging study: technical case report. Neurosurgery 48: 681-688, 2001

30. Saitoh Y, Shibata M, Hirano S, et al: Motor cortex stimulation for central and peripheral deafferentation pain. Report of eight cases. J Neurosurg 92:150-155, 2000

31. Saitoh Y, Shibata M, Sanada Y, et al: Motor cortex stimulation for phantom limb pain. Lancet 353:212, 1999

32. Tsubokawa T, Katayama Y, Yamamoto T, et al: Chronic motor cortex stimulation in patients with thalamic pain. J Neurosurg 78:393-401, 1993

33. Tsubokawa T, Katayama Y, Yamamoto T, et al: Chronic motor cortex stimulation for the treatment of central pain. Acta Neurochir Suppl 52:137-139, 1991

34. Tsubokawa T, Katayama Y, Yamamoto T, et al: Treatment of thalamic pain by chronic motor cortex stimulation. Pacing Clin Electrophysiol 14:131-134, 1991

35. Yamamoto T, Katayama Y, Hirayama T, et al: Pharmacological classification of central post-stroke pain: comparison with the results of chronic motor cortex stimulation therapy. Pain 72: 5-12, 1997

36. Yousry TA, Schmid UD, Schimdt D, et al: The central sulcal vein: a landmark for identification of the central sulcus using functional magnetic resonance imaging. J Neurosurg 85: 608-617, 1996

37. Wood CC, Spencer DD, Allison T, et al: Localization of human sensorimotor cortex during surgery by cortical surface recording of somatosensory evoked potentials. J Neurosurg 68: 99-111, 1988

Manuscript received July 17, 2001.

Accepted in final form August 9, 2001.

Address reprint requests to: Benoit Pirotte, M.D., Department of Neurosurgery, Hôpital Erasme, Université Libre de Bruxelles, 808 route de Lennik, 1070 Bruxelles, Belgium. email: bpirotte@ ulb.ac.be. 